\title{
Continuous control of spin polarization using a magnetic field
}

J. A. Gifford, G. J. Zhao, B. C. Li, Brian D. Tracy, J. Zhang, D. R. Kim, David J. Smith, and T. Y. Chen

Citation: Appl. Phys. Lett. 108, 212401 (2016); doi: 10.1063/1.4952437

View online: http://dx.doi.org/10.1063/1.4952437

View Table of Contents: http://aip.scitation.org/toc/apl/108/21

Published by the American Institute of Physics

\section{Articles you may be interested in}

Zero bias anomaly in Andreev reflection spectroscopy

Journal of Applied Physics 120, 163901 (2016); 10.1063/1.4965983

Determination of spin polarization using an unconventional iron superconductor

AIP Advances 6, 115023 (2016); 10.1063/1.4968620

Half-metallicity in highly $\mathrm{L}_{2} 1$-ordered CoFeCrAl thin films

Applied Physics Letters 109, 142410 (2016); 10.1063/1.4964464

Electric-field-induced magnetization switching in $\mathrm{CoFeB} / \mathrm{MgO}$ magnetic tunnel junctions with high junction resistance

Applied Physics Letters 108, 192406 (2016); 10.1063/1.4948763

Platinum/yttrium iron garnet inverted structures for spin current transport

Applied Physics Letters 108, 242401 (2016); 10.1063/1.4953454

Control of thermal gradient using thermoelectric coolers for study of thermal effects

Journal of Applied Physics 117, 17C508 (2015); 10.1063/1.4914542

\section{A $/ P \mid$ Applied Physics Letters}

Save your money for your research.

It's now FREE to publish with us no page, color or publication charges apply.

If your article has the

potential to shape the future of applied physics, it BELONGS in Applied Physics Letters 


\title{
Continuous control of spin polarization using a magnetic field
}

\author{
J. A. Gifford, G. J. Zhao, B. C. Li, Brian D. Tracy, J. Zhang, D. R. Kim, David J. Smith, \\ and T. Y. Chen ${ }^{\text {a) }}$ \\ Department of Physics, Arizona State University, Tempe, Arizona 85287, USA
}

(Received 8 March 2016; accepted 12 May 2016; published online 23 May 2016)

\begin{abstract}
The giant magnetoresistance (GMR) of a point contact between a $\mathrm{Co} / \mathrm{Cu}$ multilayer and a superconductor tip varies for different bias voltage. Direct measurement of spin polarization by Andreev reflection spectroscopy reveals that the GMR change is due to a change in spin polarization. This work demonstrates that the GMR structure can be utilized as a spin source and that the spin polarization can be continuously controlled by using an external magnetic field. Published by AIP Publishing. [http://dx.doi.org/10.1063/1.4952437]
\end{abstract}

Spintronic effects, such as giant magnetoresistance (GMR), ${ }^{1,2}$ tunnel magnetoresistance (TMR), ${ }^{3}$ spin-transfer torque (STT), ${ }^{4,5}$ and pure spin currents in spin Seebeck effect (SSE), ${ }^{6,7}$ are intimately related to spin of the conduction electrons. In many materials, the number of spin-up and spin-down electrons is not necessarily the same, and is measured by the spin polarization $(P)$, which is defined as the imbalance of spin-up and spin-down electrons at the Fermi level, normalized by the total number of electrons, ${ }^{8-15}$ $P \equiv \frac{N_{\uparrow}\left(E_{F}\right)-N_{\downarrow}\left(E_{F}\right)}{N_{\uparrow}\left(E_{F}\right)+N_{\downarrow}\left(E_{F}\right)}$. The value of $P$ is an intrinsic property of a material and is determined by its band structure. For example, the common magnetic metals $\mathrm{Fe}, \mathrm{Co}$, and Ni have $P$ values of about $40 \%,{ }^{8-10}$ whereas half-metals such as $\mathrm{CrO}_{2}$ can have $P$ values of close to $100 \% .^{11,12}$

Unlike conventional electronic devices which simply require a charge current, spintronics takes advantage of spinpolarized current where the $P$ value plays an important role. Materials with controllable $P$ values would be ideal to isolate the different effects of spin polarization on spintronic properties and thus reveal the true effects due to spin. However, the $P$ values of specific materials are intrinsic to those materials and cannot be easily changed. Previously, it has been shown that by doping $\mathrm{CoS}_{2}$ with $\mathrm{Fe}$, the $P$ value of the specific alloy could be tuned up to as high as $85 \% \cdot{ }^{13-15}$ It has also been proposed theoretically that the spin polarization of electrons could be tuned through double quantum dots ${ }^{16}$ or topological insulators ${ }^{17,18}$ using very large magnetic or electric fields. However, there has so far been no experimental realization of these effects, although it has recently been shown that the $P$ value of an oxide interface could be tuned by using an electric field. ${ }^{19}$ In some materials, the magnetic properties can be controlled by an electric field. ${ }^{20}$ Thus, it is nontrivial to control the $P$ value of specific materials unless their band structure is modified. Conversely, it is possible to engineer structures where the spin polarization can be tuned.

A prototype spintronic device is the GMR structure, ${ }^{1,2}$ which has been utilized in the read-head of hard drives. ${ }^{21}$ The resistance of GMR structures is low when all the magnetic layers are aligned by an external magnetic field, and it is high when the layers are aligned anti-parallel due to spindependent scattering. In this work, we show that the GMR

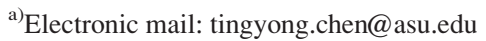

value of a point contact between a GMR structure and a superconductor can be tuned by means of a bias voltage. By directly measuring the $P$ value of the GMR structure in a varying magnetic field using Andreev reflection spectroscopy (ARS), the difference in GMR value is shown to be due to control of the spin polarization of the current in the GMR structure by an external magnetic field.

These experiments have utilized a $\mathrm{Co} / \mathrm{Cu}$ GMR structure, which has been shown previously to display very large GMR values. ${ }^{22}$ The Fe $13.5 \mathrm{~nm} /[\mathrm{Cu} 1 \mathrm{~nm} / \mathrm{Co} 1 \mathrm{~nm}]_{40}$ structure was grown by magnetron sputtering at room temperature (RT) using Fe as a buffer layer on $\mathrm{Si}[100]$ substrates. The base pressure was $2.0 \times 10^{-8}$ Torr and the sputtering gas was $\mathrm{Ar}$ at $6 \mathrm{mTorr}$. Depending on the $\mathrm{Cu}$ thickness, the Co layers can have alternative coupling: thicknesses of $1 \mathrm{~nm}$ for both $\mathrm{Cu}$ and $\mathrm{Co}$ optimize the GMR value. The largest obtained GMR value was $\sim 66 \%$ at RT and $\sim 117 \%$ at $4.5 \mathrm{~K}$, as shown in Fig. 1(a). These values are among the highest reported for polycrystalline $\mathrm{Co} / \mathrm{Cu}$ multilayers. ${ }^{22}$ Cross-section images using transmission electron microscopy revealed that the Co$\mathrm{Cu}$ layers were well-defined with strong $\{111\}$ texture in the growth direction (see Fig. 1(b)).

In this work, Andreev reflection spectroscopy (ARS) has been used to directly measure the $P$ value. At a normal metal/superconductor interface, an individual electron cannot be injected into the singlet superconductor unless it is paired with another electron with opposite spin, by reflecting a hole back into the normal metal. ${ }^{23}$ As a result, the conductance across the interface is limited by the availability of minority spins in the normal metal. For a half-metal $(P=1)$, there are no minority spins, and the conductance is 0 , while the number of minority spins for a normal metal $(P=0)$ is the same as that of the majority spins, and the conductance is 2 . Thus, the $P$ value of the metal can be determined by measuring the interface conductance. Experimentally, an interface is often not ideal and a modified Blonder-Tinkham-Klapwijk (BTK) model is often utilized to extract the $P$ value. ${ }^{24-28}$ Common superconductors such as $\mathrm{Pb}$ and $\mathrm{Nb}$ have small critical fields ${ }^{29}\left(\mathrm{H}_{\mathrm{C}}<1 \mathrm{kOe}\right)$, which could not be utilized for ARS in this work. Instead, a $0.5-\mathrm{mm} \mathrm{NbTi}$ wire was used to fabricate the superconducting tip in these experiments. NiTi wires have been previously used in superconducting magnets: ${ }^{30}$ their transition temperature is about $10 \mathrm{~K}$ with a 


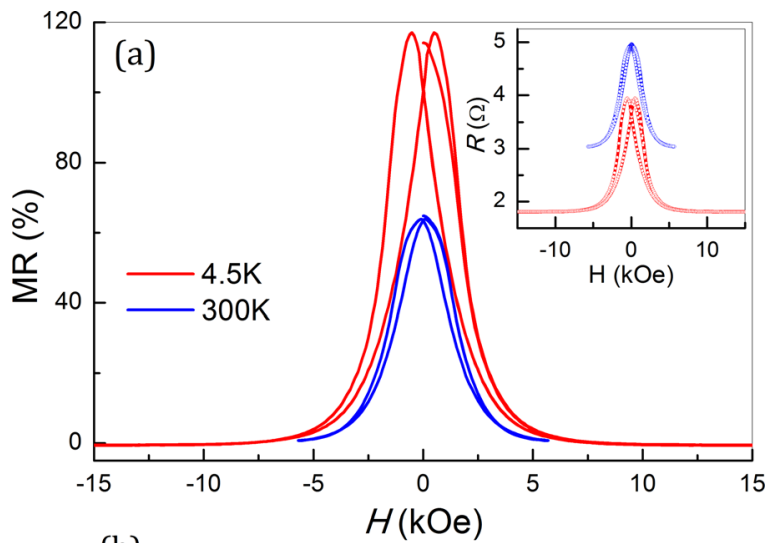

(b)

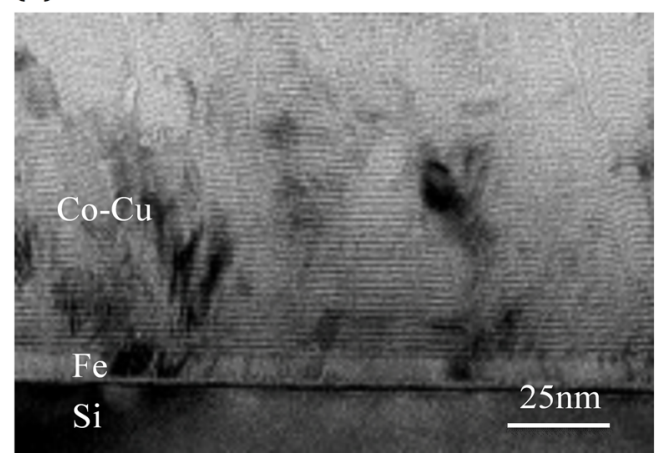

FIG. 1. (a) GMR value of sample at room temperature (blue curve) and $4.5 \mathrm{~K}$ (red curve) with raw $R$ vs. $H$ shown as inset, and (b) transmission electron microscopy cross section image of [Co $1 \mathrm{~nm} / \mathrm{Cu} 1 \mathrm{~nm}]_{40} / \mathrm{Fe} 13.5 \mathrm{~nm}$ sample.

critical field $\left(H_{C}\right)$ of $14.5 \mathrm{~T}$, which is more than sufficient for this project.

Since the sample here consists of $\mathrm{Co}$ and $\mathrm{Cu}$ multilayers, the NiTi tip is first used to measure the separate $P$ values of a $100-\mathrm{nm} \mathrm{Cu}$ layer and a $60-\mathrm{nm}$ Co layer, respectively, in a magnetic field, as shown in Figs. 2(a) and 2(b). The open circles are normalized experimental data measured at $H=0$, while the solid dots are the data measured at $H=2 \mathrm{~T}$. The raw data are shown by the inset. One can see that the external field has almost negligible effect on the normalized Andreev spectra of the $\mathrm{Cu}$ and Co layers, whereas spectrum of Co contact in field shifts slightly due to an anisotropic resistance. Most importantly, these four spectra can be welldescribed by the modified BTK model, and the extracted $P$ values are 0 for $\mathrm{Cu}$, and about $41 \%$ for $\mathrm{Co}$, which are consistent with previous studies. ${ }^{10}$ These results thus demonstrate that the NbTi tip can be utilized to determine the $P$ values, even in a magnetic field of $2 \mathrm{~T}$. It is significant that the magnetization of the 60-nm Co layer changes from $H=0$ to $H=2 \mathrm{~T}$ but not its $P$ value, which is because the point contact only contacts a region of a few nanometers across, which is often smaller than the size of a domain in the Co layer. As a result, the $P$ value does not vary when the macroscopic magnetization of the Co layer changes from zero to full saturation.

Next, a point contact is established using a NbTi tip on the $\mathrm{Co} / \mathrm{Cu}$ structures, as shown schematically by the left inset in Fig. 3. Then GMR is measured using an in-plane field with different currents of $I=0.01 \mathrm{~mA}$ and $I=1 \mathrm{~mA}$ at $4.2 \mathrm{~K}$, and the GMR value of the point contact is about $26 \%$,

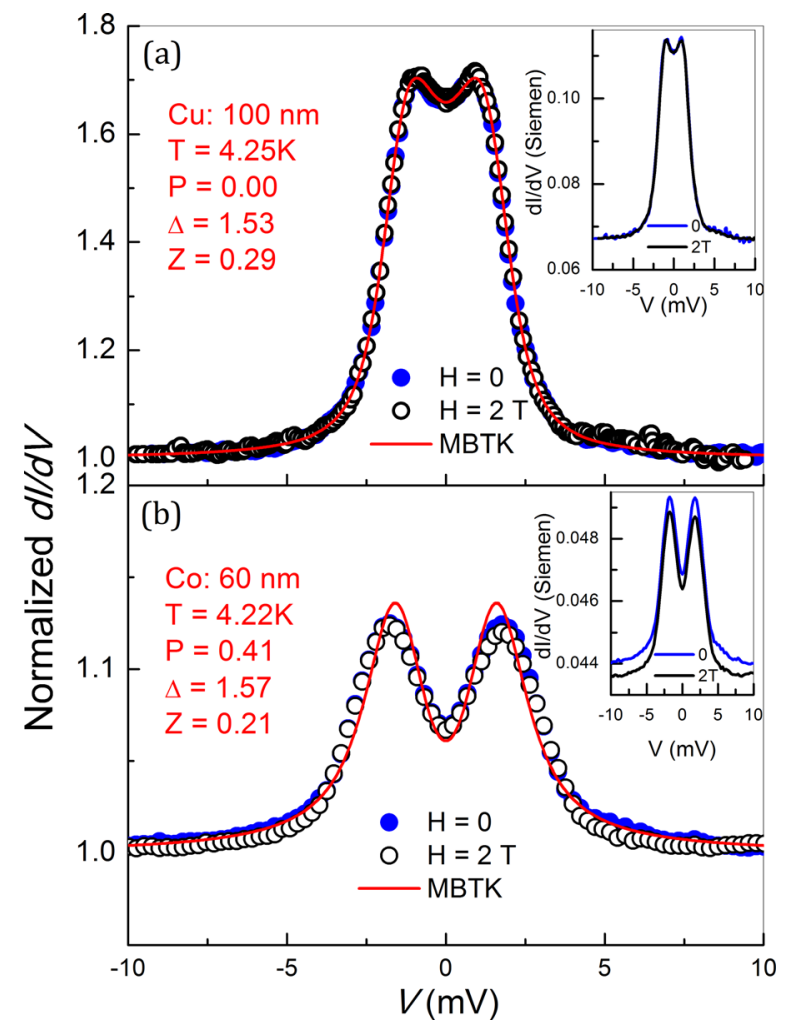

FIG. 2. (a) Normalized Andreev spectra of contacts between NiTi tip and 100-nm Cu layer at $H=0$ (solid dots) and $H=2 \mathrm{~T}$ (open circles), and (b) normalized Andreev spectra of contacts between NiTi tip and 60-nm Co layer at $H=0$ (solid dots) and $H=2 \mathrm{~T}$ (open circles). Solid curves are best fits to modified BTK model and the raw data are shown as inset.

much less than that of the sample of $117 \%$. This is because the resistance of a point contact consists of portions that do not have the same GMR value as the sample. The resistance of the point contact shifts up at $I=1 \mathrm{~mA}$, as shown by the right inset in Fig. 3. This shift is due to a larger bias voltage $(19 \mathrm{mV})$ applied on the contact for $I=1 \mathrm{~mA}$ than that $(0.24 \mathrm{mV})$ at $I=0.01 \mathrm{~mA}$. Interestingly, the GMR values of $I=0.01 \mathrm{~mA}$ and $1 \mathrm{~mA}$ have a small but clear difference, as shown in Fig. 3. The difference is about $0.68 \%$ and is from the same contact at different bias current.

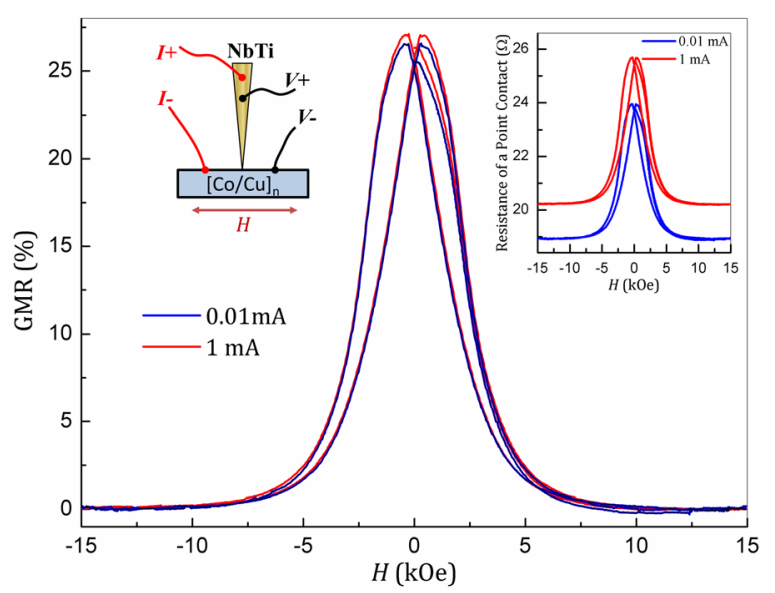

FIG. 3. GMR of point contact using NbTi tip in contact on $\mathrm{Co} / \mathrm{Cu}$ structure with currents of $0.01 \mathrm{~mA}$ (blue) and $1 \mathrm{~mA}$ (red), and schematic of point contact (left inset) and resistance of point contact at $0.01 \mathrm{~mA}$ and $1 \mathrm{~mA}$ (right inset). 
The resistance $R$ consists of the contact resistance $(R c)$, which can be affected by the Andreev reflection, and an extra resistance from the sample $(R s)$, which is not related to Andreev reflection and thus does not depend on the bias current, but has the same GMR of $117 \%$ as the sample. Rs can be estimated by the resistance from contact point to the lead, which is about $R s=0.9 \Omega$ for the contact in Fig. 3. The GMR of $R c$ can be found and the difference in the GMR of $R c$ is actually $0.83 \%$, larger than that of $R, 0.68 \%$ in Fig. 3 . Because $R c$ is larger at $I=1 \mathrm{~mA}$, any effects from $R s$ should be reduced in $R$. Furthermore, as we have shown in Fig. 2, the Andreev reflection between a NbTi tip and $\mathrm{Cu}$ or $\mathrm{Co}$ is not affected by the magnetic field at all. Therefore, a larger GMR in $R$ at $I=1 \mathrm{~mA}$ indicates a larger GMR in $R c$ than that at $0.01 \mathrm{~mA}$.

To reveal the mysterious change of GMR at different bias voltages, the Andreev spectra of the same point contact are measured at magnetic fields varying from 0 to $1.5 \mathrm{~T}$. The results are shown in Fig. 4. At each field, eight loops are measured from $-25 \mathrm{mV}$ to $+25 \mathrm{mV}$ to confirm the data: for clarification, only half of the AR spectra are plotted as a 3D plot in Fig. 4. The open circles are the experimental results and the solid curves are the best fits to the modified BTK model, ${ }^{29}$ including the extra resistance $\left(r_{E}\right) .{ }^{25}$ For fitting purposes, the data at $H=0$ are first fitted and the values of $\mathrm{Z}$ and $\Delta$ are determined using the experimental temperature. The values of $\mathrm{Z}$ and $\Delta$ are then fixed for data in fields since the data are from the same point contact, and the magnetic field will not affect the NbTi superconductor, as demonstrated in Fig. 2. The fitting parameters are discussed below. All the data can be described well by the model, as shown in Fig. 3. Since there is large GMR in the sample, the extra resistance, if any, should also have a large GMR. However, in this work, $r_{E}$ is negligible due to the small resistance of the sample. In fact, analysis of all the data shows that $r_{E}=0$. Second, in a field, if there is $r_{E}$, it becomes smaller due to the GMR effect. This leads to a higher conductance curve, ${ }^{25}$ exactly opposite to what we observed in Fig. 5(a).

The Andreev spectra at $H=0$ and $1.5 \mathrm{~T}$ in Fig. 4 seem similar but they are quite different, as shown in Fig. 5(a)

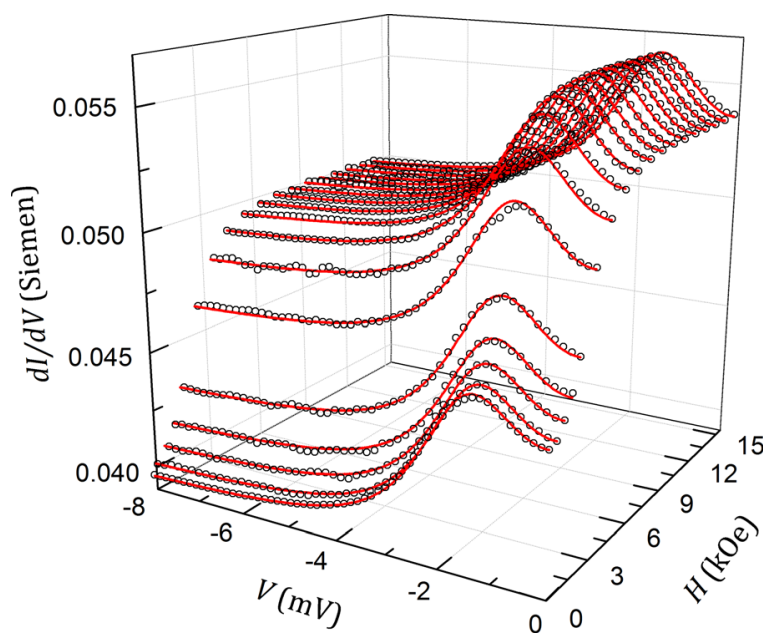

FIG. 4. Andreev spectra for NbTi tip in point contact with $\mathrm{Co} / \mathrm{Cu}$ structure in magnetic field up to $1.5 \mathrm{~T}$. Open circles are experimental data and solid curves are best fit to modified BTK model.
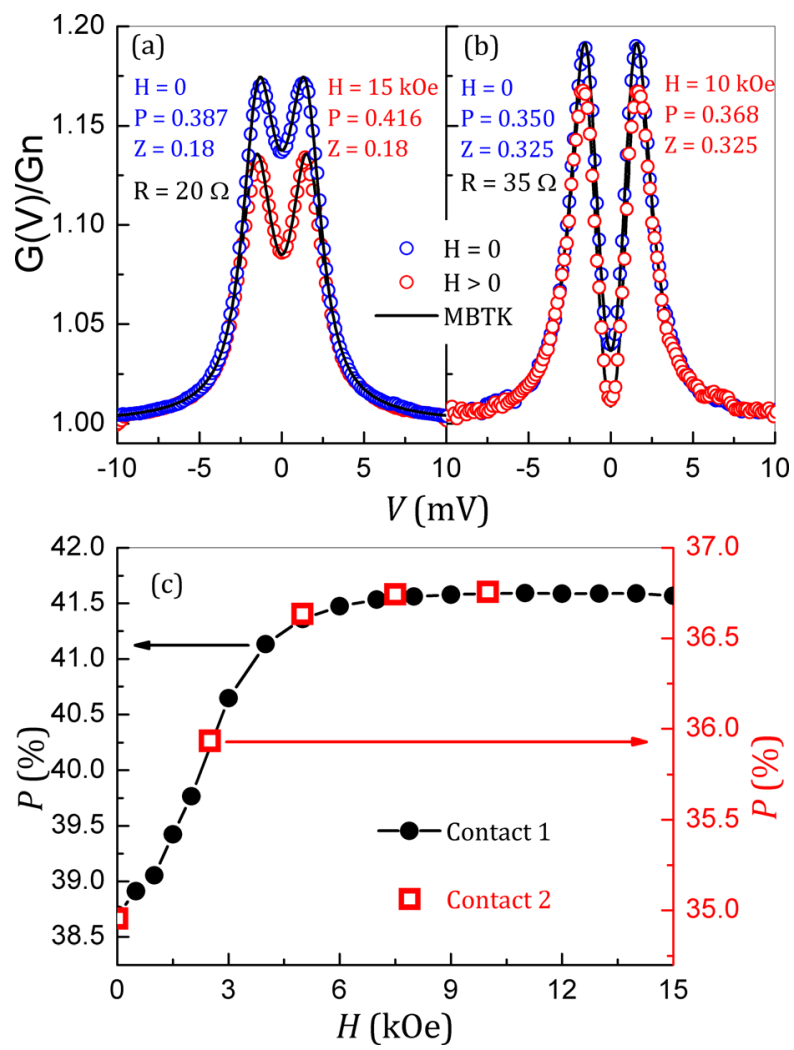

FIG. 5. (a), (b) Andreev spectra (open circles) of two point contacts at $H=0$ (blue) and $H>0$ (red) and best fit to modified BTK model (solid curves) with fitting parameters $\mathrm{T}=4.2 \mathrm{~K}, \Delta=1.55 \mathrm{meV}$, and $r_{E}=0$. (c) Spin polarization value as function of magnetic field of two point contacts.

where both are plotted together. Again, the open circles are the experimental data and the solid curves are the best fit to the modified BTK model. The difference is very clear. The AR spectrum at $1.5 \mathrm{~T}$ is lower than that at $H=0$, indicating higher $P$ value. Indeed, the $P$ value obtained at $1.5 \mathrm{~T}$ is $41.6 \%$, while the $P$ value at $H=0$ is $38.7 \%$. The difference of $2.9 \%$ in $P$ causes a clear difference in the conductance. In another contact with larger contact resistance of $35 \Omega$, higher $P$ value is again observed in a magnetic field, as shown in Fig. 5(b). The change of the $P$ value is not the same, which may be due to the difference in interfacial scattering. In fact, the $P$ value changes systematically and continuously as a function of $H$, as shown in Fig. 5(c). It increases monotonically up to 6 kOe then saturates for both contacts, following the same trend as the conductance curves at $V=0$ in Fig. 4.

The Co layers at $H=0$ form an antiferromagnetic (AFM) structure due to the RKKY interaction. ${ }^{31}$ This AFM structure scatters spin the most, causing the large GMR value. As a result, the $P$ value of $38.7 \%$ of the AFM structure will be the lowest. When all Co layers are aligned by the external magnetic field, electrons transport inside the multilayer with less scattering, resulting in the low resistance state. Hence, the measured $P$ value of $41.6 \%$ is highest. Thus, the change of the $P$ value observed from ARS is a direct measurement of the spin-dependent scattering of the GMR structure.

The different GMR values when measured at different currents in Fig. 3 can now be understood. At zero bias $(I=0.01 \mathrm{~mA})$, Andreev reflection occurs so the GMR 
includes an apparent GMR from Andreev reflection in magnetic field. Let us use the extreme case of half metal for illustration. At $H=0$, random moments scatter electron spins leading to double conductance $(P=0)$, whereas all the spins are aligned in a large field, $P=1$, and the conductance is zero. This gives a negative apparent GMR of infinity. At large bias $(V>\Delta)$, there is no Andreev reflection, nor apparent GMR. So the negative apparent GMR of Andreev reflection causes a smaller GMR at $I=0.01 \mathrm{~mA}$ in Fig. 3 .

It is interesting that the GMR of the sample is about $120 \%$, as shown in Fig. 1, but the $P$ value of the conduction electrons in Fig. 5(b) only changes by about 3\%. This may be due to the Co layers being very thin, $1 \mathrm{~nm}$, which is not enough to flip the spins. Nevertheless, the $P$ value of the structure can be continuously controlled from $38.7 \%$ to $41.6 \%$ by a modest external magnetic field, a feat that has never been achieved in any other structure. This work thus demonstrates that a GMR structure can be utilized as a spin source which can have continuously controllable spin polarization to study spintronics effects.

This work was supported as part of SHINES, an EFRC center funded by the U.S. Department of Energy, Office of Science, Basic Energy Science, under Award No. SC0012670.

${ }^{1}$ G. Binasch, P. Grünberg, F. Saurenbach, and W. Zinn, Phys. Rev. B 39, 4828(R) (1989).

${ }^{2}$ M. N. Baibich, J. M. Broto, A. Fert, F. Nguyen Van Dau, F. Petroff, P. Etienne, G. Creuzet, A. Friederich, and J. Chazelas, Phys. Rev. Lett. 61, 2472 (1988).

${ }^{3}$ J. S. Moodera, L. Kinder, T. Wong, and R. Meservey, Phys. Rev. Lett. 74, 3273 (1995).

${ }^{4}$ J. C. Slonczewski, J. Magn. Magn. Mater. 159, L1 (1996).

${ }^{5}$ L. Berger, Phys. Rev. B 54, 9353 (1996).

${ }^{6}$ D. Qu, S. Y. Huang, J. Hu, R. Wu, and C. L. Chien, Phys. Rev. Lett. 110, 067206 (2013).

${ }^{7}$ T. Kikkawa, K. Uchida, Y. Shiomi, Z. Qiu, D. Hou, D. Tian, H. Nakayama, X.-F. Jin, and E. Saitoh, Phys. Rev. Lett. 110, 067207 (2013).

${ }^{8}$ S. K. Upadhyay, A. Palanisami, R. N. Louie, and R. A. Buhrman, Phys. Rev. Lett. 81, 3247 (1998).
${ }^{9}$ R. J. Soulen, Jr., J. M. Byers, M. S. Osofsky, B. Nadgorny, T. Ambrose, S. F. Cheng, P. R. Broussard, C. T. Tanaka, J. Nowak, J. S. Moodera, A. Barry, and J. M. D. Coey, Science 282, 85 (1998).

${ }^{10}$ G. J. Strijkers, Y. Ji, F. Y. Yang, C. L. Chien, and J. M. Byers, Phys. Rev. B 63, 104510 (2001).

${ }^{11}$ Y. Ji, G. J. Strijkers, F. Y. Yang, C. L. Chien, J. M. Byers, A. Anguelouch, G. Xiao, and A. Gupta, Phys. Rev. Lett. 86, 5585 (2001).

${ }^{12}$ J. S. Parker, S. M. Watts, P. G. Ivanov, and P. Xiong, Phys. Rev. Lett. 88, 196601 (2002).

${ }^{13}$ L. Wang, K. Umemoto, R. M. Wentzcovitch, T. Y. Chen, C. L. Chien, J. G. Checkelsky, J. C. Eckert, E. D. Dahlberg, and C. Leighton, Phys. Rev. Lett. 94, 056602 (2005)

${ }^{14}$ L. Wang, T. Y. Chen, C. L. Chien, and C. Leighton, Appl. Phys. Lett. 88, 232509 (2006).

${ }^{15}$ L. Wang, T. Y. Chen, C. L. Chien, J. G. Checkelsky, J. C. Eckert, E. D. Dahlberg, K. Umemoto, R. M. Wentzcovitch, and C. Leighton, Phys. Rev. B 73, 144402 (2006).

${ }^{16}$ H. Pan, C. Wang, S.-Q. Duan, W.-D. Chu, and W. Zhang, Solid State Commun. 148, 69 (2008).

${ }^{17}$ S. S. Pershoguba and V. M. Yakovenko, Phys. Rev. B 86, 165404 (2012).

${ }^{18}$ R.-X. Zhang, H.-C. Hsu, and C.-X. Liu, e-print arXiv:1506.08242v1.

${ }^{19}$ D. Stornaiuolo, C. Cantoni, G. M. De Luca, R. Di Capua, E. Di. Gennaro, G. Ghiringhelli, B. Jouault, D. Marrè, D. Massarotti, F. Miletto Granozio, I. Pallecchi, C. Piamonteze, S. Rusponi, F. Tafuri, and M. Salluzzo, Nat. Mater. 15, 278 (2016).

${ }^{20}$ F. Matsukura, Y. Tokura, and H. Ohno, Nat. Nanotechnol. 10, 209 (2015).

${ }^{21}$ S. S. P. Parkin, U.S. patent 5585986 A (1996).

${ }^{22}$ S. S. P. Parkin, Z. G. Li, and D. J. Smith, Appl. Phys. Lett. 58, 2710 (1991).

${ }^{23}$ A. F. Andreev, Zh. Eksp. Teor. Fiz. 46, 1823 (1964) [Sov. Phys. JETP 19, 1228 (1964)].

${ }^{24}$ T. Y. Chen, Z. Tesanovic, and C. L. Chien, Phys. Rev. Lett. 109, 146602 (2012).

${ }^{25}$ T. Y. Chen, S. X. Huang, and C. L. Chien, Phys. Rev. B 81, 214444 (2010).

${ }^{26}$ P. Chalsani, S. K. Upadhyay, O. Ozatay, and R. A. Buhrman, Phys. Rev. B 75, 094417 (2007).

${ }^{27}$ G. T. Woods, R. J. Soulen, Jr., I. I. Mazin, B. Nadgorny, M. S. Osofsky, J. Sanders, H. Srikanth, W. F. Egelhoff, and R. Datla, Phys. Rev. B 70, 054416 (2004).

${ }^{28}$ G. E. Blonder, M. Tinkham, and T. M. Klapwijk, Phys. Rev. B 25, 4515 (1982).

${ }^{29}$ P. Stamenov, J. Appl. Phys. 111, $07 \mathrm{C} 519$ (2012); 113, $17 \mathrm{C} 718$ (2013).

${ }^{30}$ T. G. Berlincourt, Cryogenics 27, 283 (1987).

${ }^{31}$ S. S. P. Parkin, Phys. Rev. Lett. 67, 3598 (1991). 